\title{
ARTICLE OPEN \\ Breast cancer diagnosed during pregnancy is associated with enrichment of non-silent mutations, mismatch repair deficiency signature and mucin mutations
}

\author{
Bastien Nguyen (iD), David Venet ${ }^{1}$, Hatem A. Azim Jr ${ }^{1,2}$, David Brown (D) ${ }^{1,3}$, Christine Desmedt ${ }^{1}$, Matteo Lambertini (iD) ${ }^{1}$, Samira Majjaj ${ }^{1}$, \\ Giancarlo Pruneri ${ }^{4}$, Fedro Peccatori ${ }^{5}$, Martine Piccart ${ }^{1}$, Françoise Rothé ${ }^{1}$ and Christos Sotiriou $^{1}$
}

Breast cancer diagnosed during pregnancy $(\mathrm{BCP})$ is a rare and highly challenging disease. To investigate the impact of pregnancy on the biology of breast cancer, we conducted a comparative analysis of a cohort of BCP patients and non-pregnant control patients by integrating gene expression, copy number alterations and whole genome sequencing data. We showed that BCP exhibit unique molecular characteristics including an enrichment of non-silent mutations, a higher frequency of mutations in mucin gene family and an enrichment of mismatch repair deficiency mutational signature. This provides important insights into the biology of BCP and suggests that these features may be implicated in promoting tumor progression during pregnancy. In addition, it provides an unprecedented resource for further understanding the biology of breast cancer in young women and how pregnancy could modulate tumor biology.

npj Breast Cancer (2018)4:23; doi:10.1038/s41523-018-0077-3

\section{INTRODUCTION}

Breast cancer is the most frequently diagnosed malignancy during pregnancy. ${ }^{1}$ Its incidence is increasing given the rising trend of delayed childbearing. ${ }^{2}$ Given its rarity, few dedicated studies were performed so far; hence, our understanding of these tumors remains poor. The clinical management of these patients follows standard guidelines with only minor adaptations according to gestational age, maternal wishes and fetal considerations. ${ }^{2}$ Therefore, the molecular characterization of BCP goes beyond academic curiosity as it is of utmost clinical interest to determine if these patients should be treated similarly to non-pregnant breast cancer patients. In this report, we aimed to identify specific molecular alterations characterizing $\mathrm{BCP}$ by combining whole genome sequencing, copy number alteration and gene expression data.

\section{RESULTS}

A total of 167 patients with primary breast cancer were retrospectively included in this study, 54 of whom were diagnosed during pregnancy. Detailed patient characteristics were previously published. ${ }^{3}$ At a median follow-up of 9 years, median disease-free survival (DFS) time of BCP was 9.8 years vs. 12.5 years in controls $(P=0.041$, log rank test, Supplementary Fig. S1a). Observed 5-year overall survival (OS) rate was $95.5 \%$ vs. $85.1 \%$ in BCP and control, respectively; median OS time was not reached within the time frame of the study (Supplementary Fig. S1b). In a multivariable Cox proportional hazards regression of DFS and OS, adjusted for age at diagnosis, date of diagnosis, pathological stage and molecular subtypes by IHC, we found that BCP was associated with worse DFS (multivariable hazard ratio [mHR] $1.81 ; 95 \% \mathrm{Cl}$ 1.09-3.01, $P=0.024)$ and OS (mHR 2.53; 95\% Cl 1.20-5.36, $P=$ 0.017 ) (detailed survival data is provided in Supplementary Table S1).

BCP and controls have similar somatic copy number alteration profiles

We first sought to investigate whether tumors from BCP patients show distinct copy number alterations (CNAs) compared to tumors from matched non-pregnant breast cancer patients (controls). Hence, we performed genome-wide copy number alterations profiling on 160 formalin-fixed paraffin-embedded (FFPE) primary tumor samples from $52 \mathrm{BCP}$ patients and 108 controls. Of note, gene expression data were available for all patients as previously described. ${ }^{4}$ After quality control, CNA profiles were obtained for 125 tumor samples (78\%) from $38 \mathrm{BCP}$ and 87 controls. The main reason for exclusion was low cancer cell fraction $(\mathrm{CCF}<30 \%)$ as estimated with the Genome Alteration Print algorithm ${ }^{5}$ (Supplementary Fig. S2). No differences in clinicopathological features were observed between BCP and controls (Supplementary Table S2). We found no significant differences between BCP and controls in terms of cancer cell fraction, ploidy, and fraction of genome altered (Fig. 1a-c). Moreover, no significant differences were observed between the CNA profiles of the two groups neither at the segment nor at the chromosome arm levels, including the gains of $1 \mathrm{q}$ and $8 \mathrm{q}$ and loss

\footnotetext{
${ }^{1}$ Breast Cancer Translational Research Laboratory J.-C. Heuson, Institut Jules Bordet, Université Libre de Bruxelles (ULB), Brussels, Belgium; ${ }^{2}$ Department of Internal Medicine, American University of Beirut (AUB), Beirut, Lebanon; ${ }^{3}$ Department of Pathology, Memorial Sloan Kettering Cancer Center, New York, USA; ${ }^{4}$ Department of Pathology, IRCCS Istituto Nazionale Tumori, Milan and University of Milan, School of Medicine, Milan, Italy and ${ }^{5}$ European Institute of Oncology, Milan, Italy Correspondence: Christos Sotiriou (christos.sotiriou@bordet.be)

These authors contributed equally: Bastien Nguyen, David Venet, Hatem A. Azim.

These authors jointly supervised this work: Françoise Rothé, Christos Sotiriou.
}

Received: 23 March 2018 Revised: 4 July 2018 Accepted: 9 July 2018

Published online: 06 August 2018 

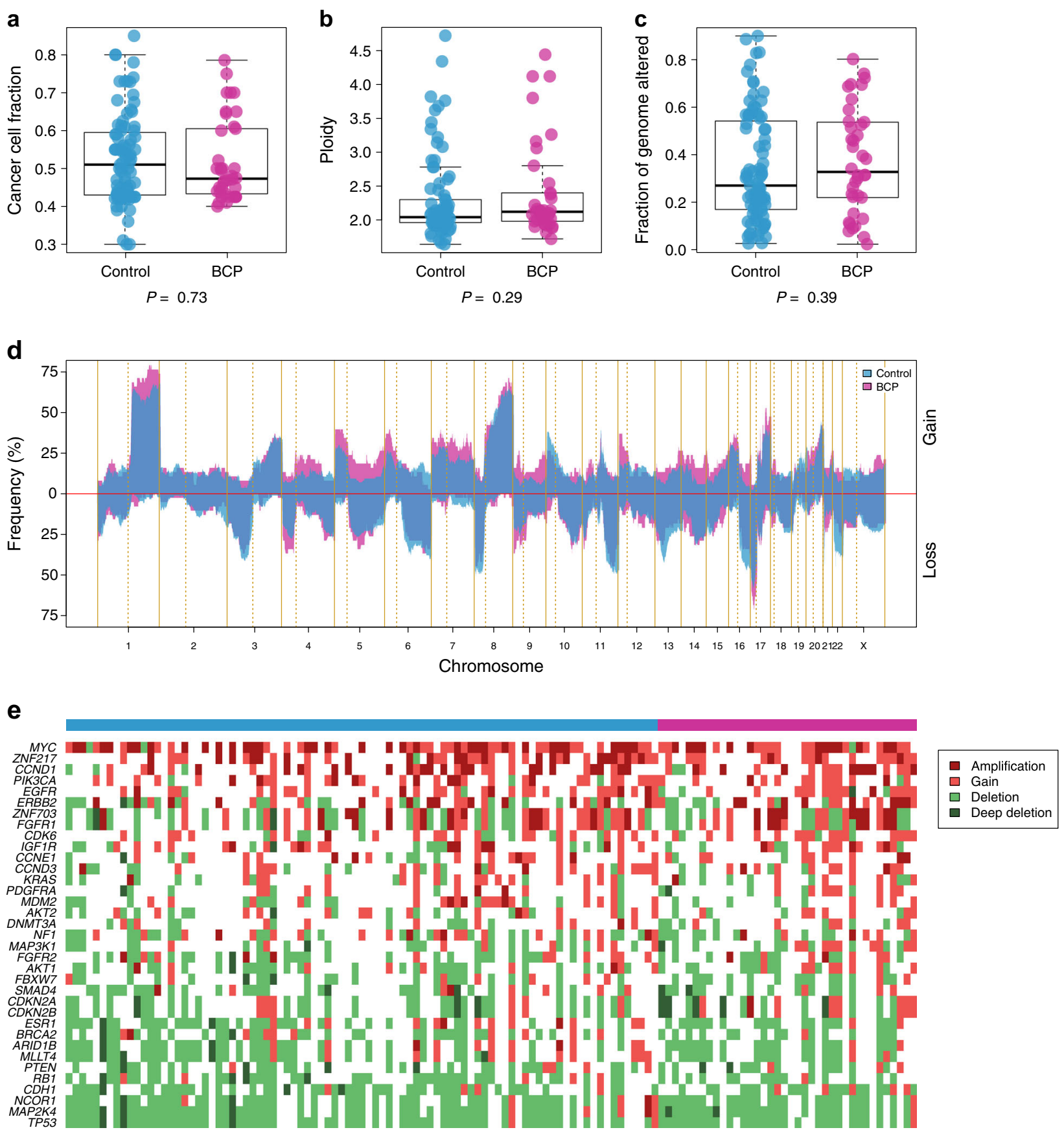

Fig. 1 Summary of the genome-wide copy number analysis of 87 controls and 38 BCP tumor samples. a-c Comparison of cancer cell fraction, ploidy and fraction of genome altered between controls and BCP. $\mathbf{d}$ Comparison of the CNA frequencies of controls (blue) and BCP (pink). e Heatmap of 35 CNA breast cancer driver genes according to their alterations; controls (blue) and BCP (pink). $P, p$-value derived for the Mann-Whitney $U$ test

of $8 p$, reported to frequently occur in breast cancer ${ }^{6}$ (Fig. 1d). We also compared CNAs profiles by intrinsic subtypes as defined by PAM50 and found no significant differences (Supplementary Fig. S3 and Supplementary Table S3).

We next focused our analysis on the 35 genes that were previously identified as CNA drivers in breast cancer. ${ }^{7}$ As expected, $M Y C$ oncogene was the most frequently gained/amplified whereas TP53 tumor suppressor gene was the most frequently lost/deleted across the whole cohort (Fig. 1e). Using GISTIC2.0, ${ }^{8}$ we identified 22 focal amplifications and 23 focal deletions and found no differences between their prevalence in the two groups
(Supplementary Fig. S4). Taken together, these results suggest that the CNA profiles of BCP and controls are similar.

$\mathrm{BCP}$ shows a higher number of non-silent mutations

To identify potential genomic differences between BCP patients and controls, we performed whole genome sequencing (WGS) on paired DNA samples extracted from FFPE blocks (i.e., primary tumors and histologically normal axillary lymph nodes) in a subset of 53 breast cancer patients from our initial series, 35 of whom were BCP (Supplementary Fig. S2 and Supplementary Table S2). We achieved $32 \mathrm{X}$ and 19X median haploid genome coverage for 


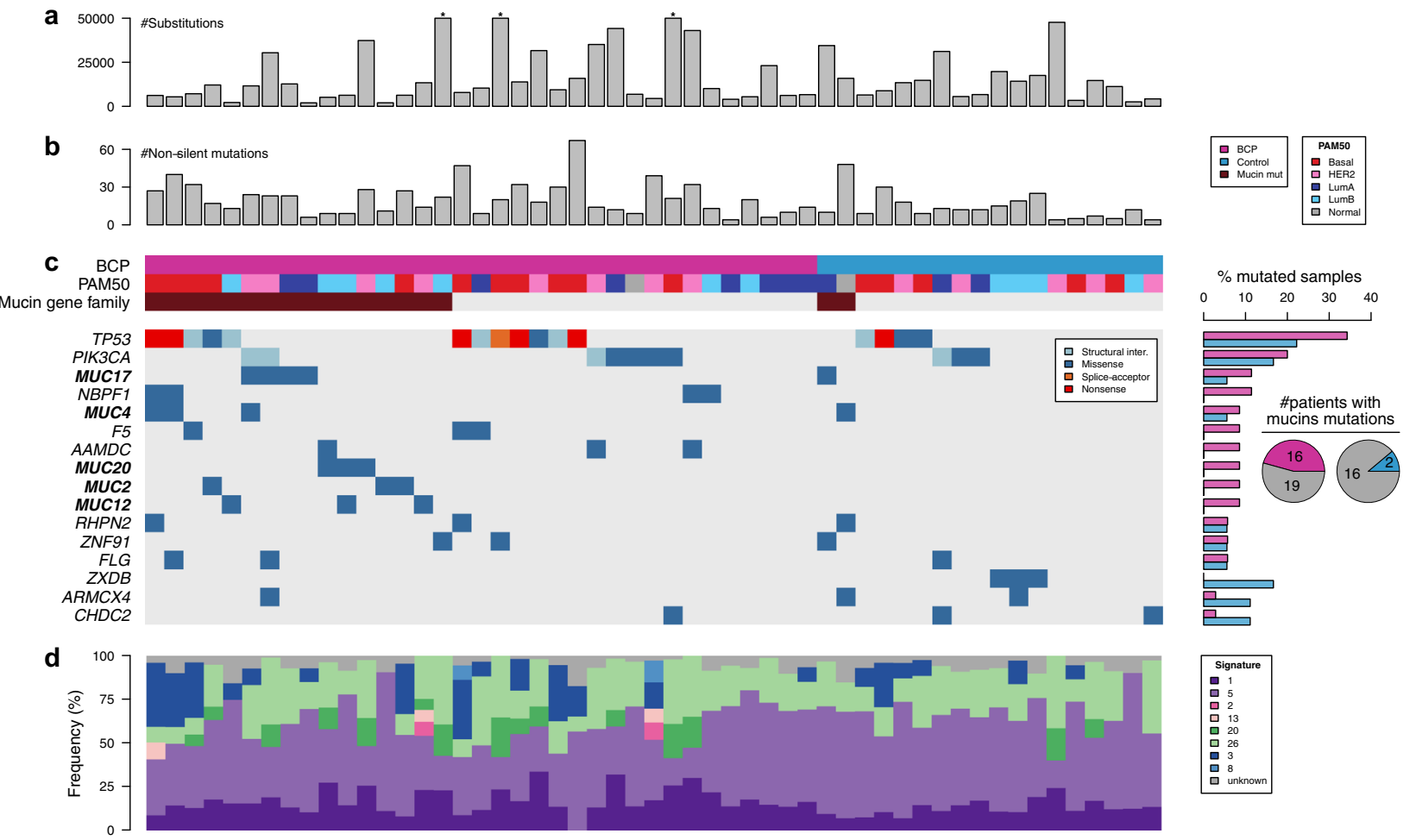

Fig. 2 Mutational landscape of individual BCP and controls. a Bar chart representing the absolute number of substitutions in BCP and controls, $y$-axis limited to 50,000 indicated by $\left(^{*}\right)$. b Bar chart representing the absolute number of non-silent mutations in BCP and controls (median: 20 vs. $12, P=0.027$, respectively). c Co-mutation plot showing genes harboring at least one non-silent mutation with a frequency of at least 5\% across the whole cohort, and their corresponding frequencies in BCP and controls (right). d Proportion of breast cancer substitution signatures in each sample. Signatures are colored according to broad biological groups: 1 and 5 are associated with clock-like processes, 2 and 13 are APOBEC-related, 20 and 26 are associated with mismatch-repair deficiency, 3 and 8 are associated with homologousrecombination deficiency

tumor and normal samples respectively, which is similar in range to previous studies ${ }^{7}$ (Supplementary Fig. S5). We detected a median of 13,829 and 10,084 single nucleotide variants (SNVs) and a median of 21 and 26 small insertions and deletions (Indels) in BCP and controls, respectively, and found no difference between the two groups (Fig. 2a and Supplementary Fig. S6a-c). Moreover, there was no difference in structural variations (insertions, deletions, duplications) nor tumor heterogeneity as assessed by the MATH score ${ }^{9}$ (Supplementary Fig. S6d-f).

We identified a median of 14 non-silent mutations per tumor which is comparable to another large-scale breast cancer cohort study $^{7}$ (Supplementary Table S4). Interestingly, BCP had a significantly higher number of non-silent mutations than controls (median: 20 vs. $12, P=0.027$, Fig. $2 \mathrm{~b}$ and Supplementary Fig. S6gh). This observation remained consistent after correcting for potential confounding factors including age at diagnosis, date of diagnosis, pathological stage and molecular subtypes by $\mathrm{IHC}(P=$ 0.019 , Fig. $\mathrm{S6g}$ ). Compared to controls, BCP had also a significantly higher number of mutations previously reported in breast cancer in the Catalog of Somatic Mutations in Cancer (COSMIC) database $^{10}(P=0.018$, Supplementary Fig. S6i). At the gene level, we identified 17 genes harboring at least one non-silent mutation with a frequency of at least $5 \%$ across all patients. Of those, TP53 and PIK3CA were the most frequently mutated genes without any significant difference between the two groups (Fig. 2c).

BCP is associated with a higher frequency of mutations in mucin gene family

MUC17 was the third most mutated gene and four other mucin gene family members namely MUC2, MUC4, MUC12, and MUC20, were among the most frequently mutated genes in BCP (Fig. 2C).
Within the mucin gene family, we identified 20 missense mutations and one nonsense mutation in BCP compared to only two missense mutations in controls. Among these 20 mucin variants, 10 were present in the COSMIC database, ${ }^{10}$ which was higher than expected by chance $(P=0.006$, Monte-Carlo test, Supplementary Table S4). Altogether, we found a significantly higher number of BCP with non-silent mutations in the mucin gene family compared to controls (45.7 vs. $11.1 \%$ respectively, $P=$ 0.015, Fig. 2c). This observation remained consistent after correcting for classical clinicopathological features $(P=0.008)$. Similar findings were observed by comparing BCP with 56 matched controls taken from the TCGA dataset (45.7 vs. $23.1 \%$ respectively, $P=0.034$ ). Acknowledging that some mucins (MUC4, MUC16) are known to give rise to false positive calls due to technical artifacts, ${ }^{11}$ we removed these two genes and confirmed the above-mentioned results ( 37.1 vs. $5.5 \%, P=0.020$ and 37.1 vs. $14.3 \%, P=0.020$, using controls and TCGA controls, respectively).

We did not find any differences in clinicopathological features or survival according to mucin mutational status (Supplementary Table S5 and Supplementary Fig. S7). There were three hotspots mutations (i.e., present in two distinct patients) two in MUC17 and one in MUC20, and five missense mutations were clustered within 260 base pairs of MUC2 (Fig. 3a). None of these mutations were in annotated protein domains. Since the glycosylation of mucins is known to play a major role in producing a chemical barrier at the epithelium of tubular organs for protection and lubrication, we interrogated whether these mutations could affect glycosylation acceptor sites. The mucin O-glycosylation is characterized by the addition of $\mathrm{N}$-acetylgalactosamine (GalNAc) to the hydroxyl group of serine or threonine residues. ${ }^{12}$ Remarkably, $40.9 \%$ of missense mutations affecting mucins resulted in an amino acid change to a 
a MUC2
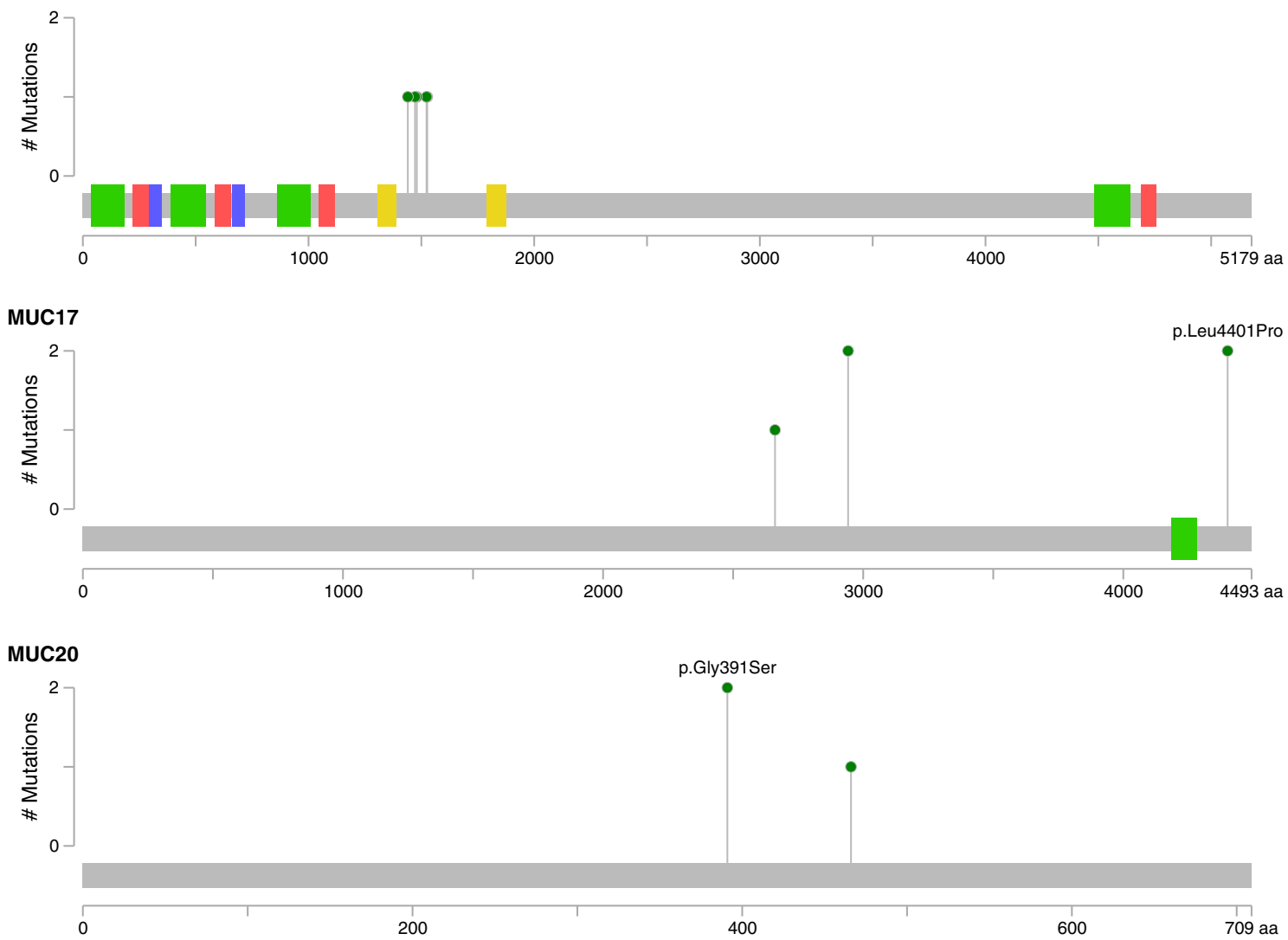

b

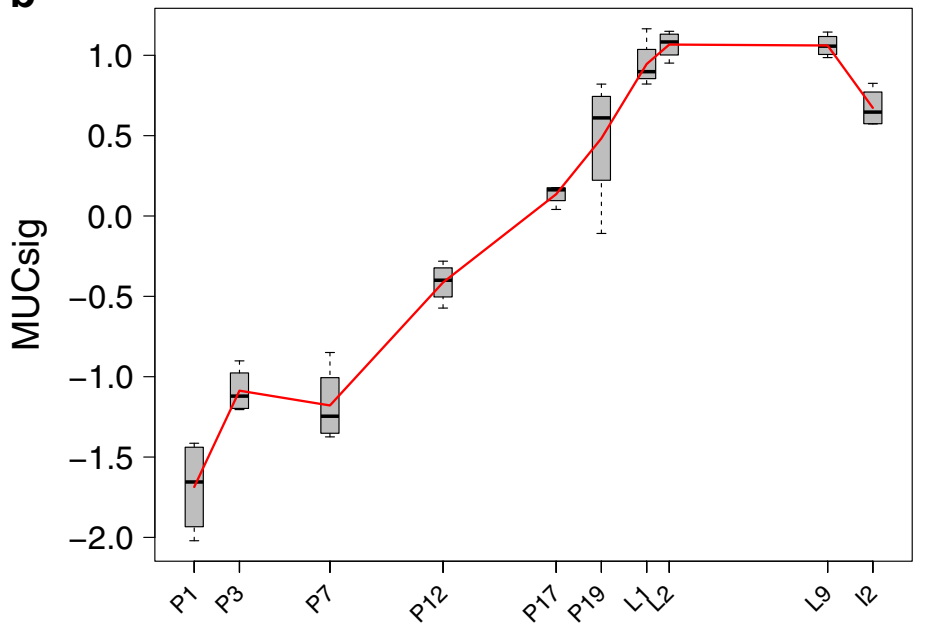

Development time
C

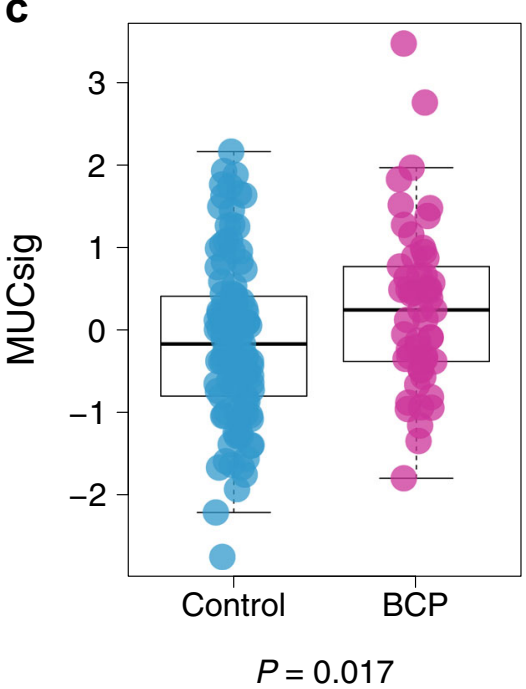

Fig. 3 Enrichment of mucin mutations and upregulation in BCP. a Lollipop plots were generated using cBioPortal Mutation Mapper. Each lollipop denotes a unique missense mutation for MUC2, MUC17, and MUC20 in BCP. b MUCsig according to normal adult mouse mammary development (from pregnancy day 1 to involution day 2). c Comparison of MUCsig between controls and BCP. $P, p$-value derived for the Mann-Whitney $U$ test

serine residue, which was significantly higher than expected by chance ( $P=0.0002$, Monte-Carlo test), suggesting mucin hyperglycosylation in BCP. We also found that the frequency of missense mutations resulting in a gain of serine site in mucins in the TCGA dataset was significantly lower compared with BCP $(6.3 \%$ in TCGA vs. $40.9 \%$ in $B C P, P<0.001)$. Since mucins expression is known to increase throughout gestation in mice, ${ }^{13}$ we expected that mucins were also upregulated in BCP. We therefore derived a metagene signature comprising all members of the mucin gene family (called "MUCsig") from the corresponding gene expression data and found higher expression of MUCsig in BCP than in controls $(P=0.017$, Fig. 3b-c). Altogether, these results show that BCP is associated with an increased expression of mucins as well as a higher frequency of mutations in mucin 
a

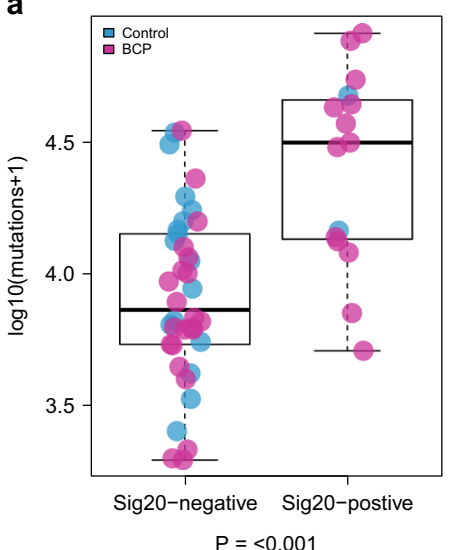

b

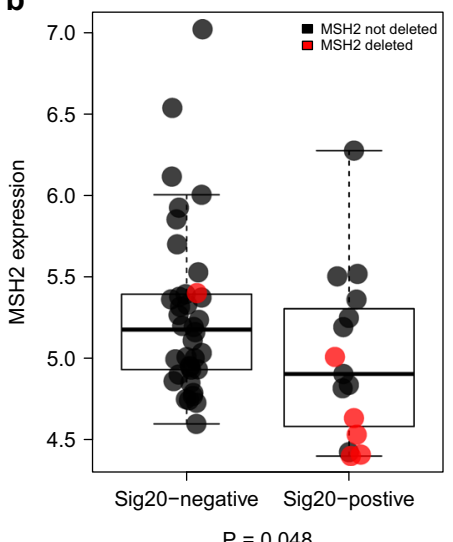

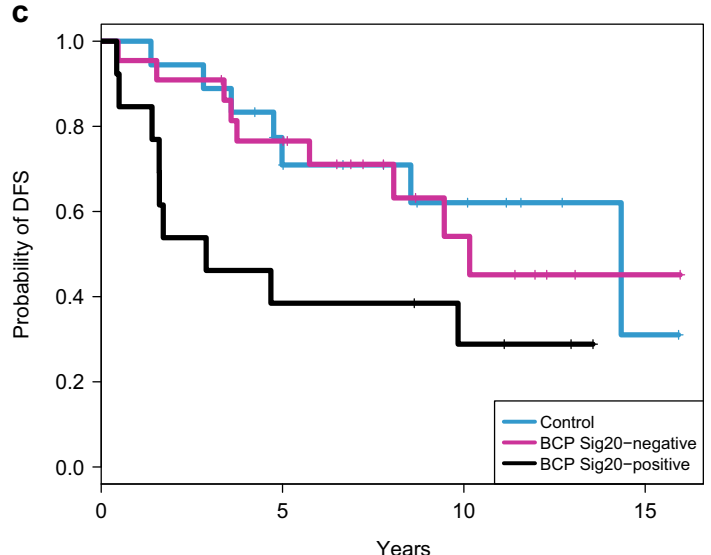

Fig. 4 Association of signature 20 with mutational load and clinical outcome. a Comparison of SNV mutational load between Sig20 negative and Sig20 positive tumors. b Comparison of MSH2 expression between Sig20 negative and Sig20 positive tumors. c Kaplan-Meier plot showing the difference in DFS between control patients $(N=18)$, BCP patients with Sig20 negative tumors $(N=22)$ and BCP patients with Sig20 positive tumors $(N=13) . P, p$-value derived for the Mann-Whitney $U$ test

gene family that may potentially lead to mucin hyperglycosylation.

$\mathrm{BCP}$ is enriched in mutational signature related to mismatch repair deficiency

To have a better understanding of the etiology of $B C P$, we interrogated the contribution of base-substitution signatures known to occur in breast cancer. ${ }^{7}$ When evaluating the proportion of each signature present in each sample, we found that signature 1 was more prevalent in BCP compared to controls whereas signature 5 was more prevalent in controls $(P=0.013, \mathrm{FDR}=0.053$ and $P=0.01, F D R=0.053$, respectively, Fig. $2 \mathrm{~d}$ ). These results remained consistent after controlling for clinicopathological features $(P=0.002, \mathrm{FDR}=0.014$ and $P=0.004, \mathrm{FDR}=0.016$, respectively). When evaluating the presence or absence of mutational signatures we found that signature 20 (Sig20) was found in 13 out of $35 \mathrm{BCP}(37.1 \%)$, as compared to only 2 out of 18 controls $(11.1 \%)(P=0.059, \mathrm{FDR}=0.410$, Fig. $2 \mathrm{~d})$. When controlling for clinicopathological features, this observation was significant $(P=0.004, \mathrm{FDR}=0.029)$. Signature 1 is known to be associated with age at diagnosis while the etiology of signature 5 is still unclear. Sig20, previously found in stomach and breast cancers, is related to DNA mismatch repair (MMR) deficiency. ${ }^{14}$ Of interest, this signature remained significantly enriched in $\mathrm{BCP}$ when increasing the number of controls with 64 matched cases derived from the BRCA560 dataset (37.1 vs. $3.1 \%, P<0.001)$. No classical clinicopathological features were associated with $\mathrm{BCP}$ Sig20-positive tumors except progesterone receptor negative status (Supplementary Table S6). We found that Sig20 frequency was strongly correlated with SNV mutational load $(\rho=0.56, P<$ 0.001 , Supplementary Fig. S8a) with Sig20-positive tumors harboring a median of $31,632 \mathrm{SNVs}$, as compared to 7,352 SNVs in Sig20-negative tumors $(P<0.001$, Fig. 4a). Next, we interrogated if Sig20 could be caused by alteration of genes involved in the MMR machinery either at the expression or copy number levels. The first step of MMR is the recognition of replication errors mediated by MutS homolog complexes; $\mathrm{MSH} 2$ and $\mathrm{MSH}^{15}{ }^{15}$ We found a significantly lower expression of $M S H 2$ in patients harboring Sig20 ( $P=0.047$, Fig. $4 \mathrm{~b})$ corroborated by a negative correlation between $M S H 2$ expression and Sig20 frequency $(\rho=$ $-0.27, P=0.024$, Supplementary Fig. S8b). This could be partially caused by CNA in MSH2 since 5 out of 15 Sig20-positive versus 1 out of 38 Sig20-negative tumors harbored MSH2 deletions (33.3 vs. $2.6 \%, P=0.01$ ). Finally, we interrogated the impact of Sig20 on survival and found that BCP Sig20-positive patients had a shorter
DFS than BCP Sig20-negative patients (median DFS time of 2.9 years vs. 10.2 years respectively $P=0.091$, log rank test, Fig. 4c). In Sig20-positive patients the median OS was 6.72 years while the median OS was not reached in BCP Sig20-negative patients $(P=$ 0.009, log rank test, Supplementary Fig. S9). This was not significant in a multivariate model (DFS mHR 1.06; $95 \% \mathrm{Cl}$ $0.21-4.27, P=0.31$; OS mHR 0.8; $95 \% \mathrm{Cl} 0.12-5.07, P=0.81$, respectively). Overall, these results suggest that some BCP patients show a defective MMR due to copy number loss of $M S H 2$.

\section{DISCUSSION}

This study reveals important molecular differences characterizing $\mathrm{BCP}$ that may potentially represent a biologic explanation for their rather aggressive clinical behavior. First, BCP was enriched in nonsilent mutations that could have potential oncogenic functiond. Second, $45 \%$ of BCP harbored a mutation in mucin gene family in addition to an upregulation of mucins at the mRNA level. Like in mice, ${ }^{13}$ this could be due to physiological change induced by pregnancy to prepare the breast for lactation. Our hypothesis is that some preexisting subclones carrying mucin mutations could have a growth advantage under pregnancy state. Another argument in favor of this hypothesis is the fact that most mucin mutations resulted in an amino acid change to a serine residue and that some of them are in hotspot regions. It has been previously found that in breast cancer, alterations in mucin expression or glycosylation influence tumor growth, adhesion, invasion, and immune surveillance. ${ }^{16,17}$ The impact of missense mutations resulting in an amino acid change to a serine residue on the glycosylation status of mucins is unknown, but it is tempting to speculate that these alterations could influence their function, stability and secretion. More investigations are required to determine the exact effect of mucin mutations in BCP and in breast cancer in general, but these alterations could play a role in BCP biology.

Moreover, BCP showed a higher prevalence of signatures 1 and 20 and a lower prevalence of signature 5 . The etiology of signature 5 is not well understood. ${ }^{18}$ The high prevalence of signature 1 cannot be explained by a difference in age at diagnosis or age of the blocks since similar results were found in a multivariate analysis after adjusting for both variables. $37.1 \%$ of BCP were associated with signature 20 (Sig20), attributable to DNA mismatch repair deficiency. This is surprising given the low frequency $(1-2 \%)$ of MMR deficiency recently reported in breast cancer. ${ }^{19}$ Mechanistically, this could be explained in part by the deletion of $M S H 2$, a key gene involved in MMR. Survival analysis 
showed that BCP Sig20-positive patients had the worst prognosis whereas BCP Sig20-negative patients had DFS comparable to controls. MMR deficiency and high mutational burden have been shown to predict clinical benefit to immune checkpoint blockade in colorectal and other types of highly immunogenic cancers. ${ }^{20,21}$ To date, the role of checkpoint inhibitors in the treatment of breast cancer is under intensive investigation and the results are still awaited. ${ }^{22}$ While the feasibility of investigating new agents in such peculiar disease is rather complex, these results could potentially open the door to identify high-risk BCP patients who could benefit from immunotherapy.

A potential limitation of our study is that we used archived FFPE samples that are known to be challenging for WGS due to DNA degradation and induction of artefacts. Indeed, the higher proportion of signature 1 and 5 observed in our study could be due to $C>T$ artefacts induced by formalin fixation. Nonetheless, BCP and controls were processed in the same way with no difference in the age of the blocks and the sequencing coverage reached in normal and in tumor tissues was comparable to other studies. $^{7}$ Another limitation of our study is the lack of epigenetic profiling analysis. As it is known that pregnancy induce epigenetic changes in epithelial cells to support mammary development, ${ }^{23}$ we can hypothesize that these modifications could impact breast cancer biology. Therefore, the study of such modifications in BCP is worthy further investigation. In conclusion, we believe that our work provides important insights into the biology of BCP and a unique resource to study the biology of breast cancer in young women and how pregnancy could modulate tumor biology.

\section{METHODS}

\section{Patients and samples}

A total of 167 patients with primary breast cancer were retrospectively included in this study, 54 of whom were diagnosed during pregnancy. All patients were diagnosed and followed up at the European Institute of Oncology (IEO, Milan, Italy) from 1996 to 2010 . As previously described, ${ }^{3}$ this is a case-control study, in which pregnant breast cancer patients and controls were matched according to age, tumor size, nodal status, and date of diagnosis. For the current genomic analysis, we opted to exclude patients who received neoadjuvant therapy to avoid potential impact of treatment on the obtained results. The majority were treated with anthracycline-based regimen (individual patients data are presented in Supplementary Table S1). All patients had available FFPE tissue from the primary tumor resection and there was only one tumor sample per patient. All control patients were pre-menopausal at time of diagnosis. ER/PR-status were defined by ASCO-CAP. For the classification of Luminal A and B we used a cut-off of Ki67 > 20\% according to the St Gallen 2015 Consensus Meetings. ${ }^{24}$ Matched normal tissues were collected from histologically confirmed tumor-free axillary lymph nodes or tumor-adjacent normal tissue and there was only one normal sample per patient. FFPE tissue sections were deparaffinized by xylene followed by a $100 \%$ ethanol wash. DNA extraction was performed using the QIAamp DNA FFPE Tissue Kit (Qiagen, Hilden, Germany) following the manufacturer's recommendations. The quantity of double-stranded DNA was evaluated using the Qubit dsDNA BR Assay Kit. For the WGS, we selected 18 control patients based on major clinicopathological features of the $35 \mathrm{BCP}$ patients, namely age at diagnosis, ER status, and grade. All patients provided written informed consent for the use of tissue samples for research purposes as per the IEO institutional policies. This study was approved by the Ethics Committee of Institut Jules Bordet (Number 1782). The validation of the enrichment of mutations in the mucin family genes in BCP were done by comparing the frequency of these mutations in BCP patients with putatively non-pregnant patients retrieved from the TCGA dataset ${ }^{25}$ and selected to have similar age, estrogen receptor (ER) and progesterone receptor (PR) distribution ( $N$ =56) (Supplementary Table S7). The validation of the enrichment of signature 20 in BCP were done by comparing the frequency of this signature in $\mathrm{BCP}$ patients with putatively non-pregnant patients retrieved from the 560 breast cancer dataset ${ }^{7}$ (referred to as BRCA560) and selected to have similar age, ER and PR distribution $(N=64)$.

\section{Transcriptomic profiling}

All samples were hybridized on Affymetrix Human Genome U219 array plates following the manufacturer's protocol, as described before. ${ }^{4}$ The metagene signature MUCsig was calculated by taking the mean expression level of all genes present in the mucin family, scaled to a standard deviation of one and centered around zero. The publicly available murine data set derived from normal breast of pregnant mice (GEO ID: GSE8191 ${ }^{13}$ ) was used to evaluate mucin expression in the normal breast during pregnancy. Ensembl database was used to convert mouse gene names to the human equivalent.

\section{Genome-wide copy number analysis}

Hematoxylin and eosin slides from the archived FFPE blocks were reviewed by a pathologist (G.P.) to confirm diagnosis and evaluate tumor content. Samples with tumor purity below $60 \%$ were macrodissected $(N=56)$. DNA was extracted as described above. A total of $80 \mathrm{ng}$ of DNA was used for copy number profiling using the Affymetrix OncoScan ${ }^{\circledast}$ FFPE Assay Kit according to the manufacturer's instructions. The raw intensity values from the scanned chips were normalized to obtain Log2 ratios, B allele frequencies and genotyping calls (AA/AB/BB) using Affymetrix Power Tools. We used release NA.33 of the NetAffx library for the reference model and annotation. We computed the median absolute pairwise deviation and the median auto-correlation from the normalized log2 ratios as quality control metrics and used a threshold of 0.30 and 0.5 , respectively, to flag failed arrays. Further details are provided in the Supplementary Methods.

\section{Library preparation and whole genome sequencing}

For each of 53 patients, two samples of $1 \mu \mathrm{g}$ genomic DNA from tumor and histologically normal axillary lymph nodes were whole genome sequenced at The McDonnell Genome Institute at Washington University (St Louis MO, USA) on an Illumina HiSeqX platform. Briefly, manual dual indexed libraries were constructed with $1 \mu \mathrm{g}$ of FFPE genomic DNA for the 53 tumor/normal pairs using the Accel-NGS 2S Plus Library Kit (Swift, MI, USA). Samples were fragmented on the Covaris LE220 instrument with 350bp target insert size. PCR cycle optimization was performed to prevent over-amplification of the libraries. The concentration of each library was determined through qPCR (Kapa Biosystems, MA, USA). For the normal samples, each library was loaded on one lane of a HiSeqX flow cell, whereas for tumor samples, each library was loaded across two lanes of a HiSeqX flow cell. $2 \times 150$ paired-end sequence data were generated at a target depth of $30 \times$ (normal) and 60 (tumor) haploid genome coverage. All sequencing data are available in EGA under accession "EGAS00001002685". Further details are provided in the Supplementary Methods.

\section{Tumor heterogeneity}

To quantify the level of intra-tumor heterogeneity present in a sample, we used the MATH score as previously described ${ }^{9}$;

$M A T H=\frac{M A D(V A F s)}{\operatorname{median}(V A F s)}$

where $M A D(V A F s)$ is the median absolute deviation of the variant allele fractions (VAFs) of all the mutations (coding and noncoding) in a tumor.

\section{Mutational signature}

All samples were analyzed using deconstructSigs ${ }^{26}$ to extract signatures based on the Wellcome Trust Sanger Institute Mutational Signature Framework.

\section{Statistical analysis and survival analysis}

Except for age and date of diagnosis that were considered as continuous variables and therefore compared using the non-parametric Mann-Whitney $U$ test, differences in other clinicopathological characteristics between BCP and controls were analyzed using the $x^{2}$ test or the Fisher exact test when appropriate. All statistical tests comparing BCP and controls were done using the non-parametric Mann-Whitney $U$ test and the Fisher exact test for continuous and categorical variables, respectively. Independent association between continuous and binary variables with $B C P$ vs. controls was investigated using linear and logistic regressions, respectively. All multivariate tests were adjusted for age at diagnosis, date of diagnosis, pathological stage, and molecular subtypes by IHC. All 
interaction and multivariate tests were done using analysis of variance to compare the models with and without the extra term.

All correlations were measured using the non-parametric Spearman's rho coefficient. Reported $P$-values were two-tailed, and differences were considered significant when the $P$-value was less than 0.05 . When applicable, multiple testing correction was done using the false discovery rate method (FDR), ${ }^{27}$ FDR below 0.05 being considered significant. All analyses were done in R software version 3.3.2 (available at www.r-project. org) and Bioconductor version 3.4.

Survival endpoint was DFS and calculated from the date of surgery to any loco-regional or distant recurrence, contralateral BC, other primary tumor or death from any cause, whichever occurred first. In the absence of any of the above-mentioned events, survival was censored at the last follow-up visit or phone call with the patient. Survival curves were estimated using the Kaplan-Meier method and compared by the log-rank test. The prognostic impact of pregnancy on survival was evaluated using univariate and multivariate Cox proportional hazards regression models and expressed as hazard ratio (HR) with $95 \% \mathrm{Cl}$. Multivariate analysis was adjusted for standard clinical prognostic factors (age at diagnosis, date of diagnosis, pathological stage, and molecular subtypes by $\mathrm{IHC}$ ). Further details are provided in the Supplementary Methods.

\section{Data availability}

Raw gene expression data, together with patients' characteristics, are publicly available on GEO http://www.ncbi.nlm.nih.gov/geo/, under accession number GSE53031. Sequencing data have been deposited at the European Genome-Phenome Archive (http://www.ebi.ac.uk/ega/), under accession number EGAS00001002685.

\section{ACKNOWLEDGEMENTS}

We thank the patients who contributed to this study by providing tissue samples and acknowledge the clinical staff at European Institute of Oncology for their dedication. We acknowledge whole genome sequencing data generation by the production sequencing group at the McDonnell Genome Institute at Washington University in St. Louis, and the oversight of this work by Elaine Mardis.

\section{AUTHOR CONTRIBUTIONS}

Conceptualization, B.N., H.A., and C.S.; Methodology, B.N., D.V., and D.B.; Formal Analysis, B.N., D.V., and D.B.; Investigation, B.N. and S.M.; Resources, G.P. and F.P.; Writing-Original Draft, B.N.; Writing-Review \& Editing, B.N., D.V., F.R., C.D., M.L, D.B., H.A., M.P., and C.S., Funding Acquisition, F.R., H.A., M.P and C.S., Supervision, F.R., H.A and C.S. All authors reviewed and approved the final manuscript. The work was supported by grants from the Breast Cancer Research Foundation (BCRF). C. Sotiriou is a ULB Professor funded by the Fonds National de la Recherche Scientifique (FNRS). M Lambertini is supported by an ESMO Translational Research Fellowship. B. Nguyen is supported by a Télévie fellowship.

\section{ADDITIONAL INFORMATION}

Supplementary information accompanies the paper on the npj Breast Cancer website (https://doi.org/10.1038/s41523-018-0077-3).

Competing interests: The authors declare no competing interests.

Publisher's note: Springer Nature remains neutral with regard to jurisdictional claims in published maps and institutional affiliations.

\section{REFERENCES}

1. Anderson, J. M. Mammary cancers and pregnancy. Br. Med. J. 1, 1124-1127 (1979).

2. Loibl, S. et al. Breast cancer diagnosed during pregnancy. JAMA Oncol. 1, 1145 (2015).

3. Azim, H. A. et al. The biological features and prognosis of breast cancer diagnosed during pregnancy: a case-control study. Acta Oncol. 51, 653-661 (2012).

4. Azim, H. A. et al. Biology of breast cancer during pregnancy using genomic profiling. Endocr. Relat. Cancer 21, 545-554 (2014).

5. Popova, T. et al. Genome Alteration Print (GAP): a tool to visualize and mine complex cancer genomic profiles obtained by SNP arrays. Genome Biol. 10, R128 (2009).

6. Mika, T. et al. Molecular cytogenetics of primary breast cancer by CGH. Genes Chromosom. Cancer 21, 177-184 (1998).

7. Nik-Zainal, S. et al. Landscape of somatic mutations in 560 breast cancer wholegenome sequences. Nature 534, 1-20 (2016).

8. Mermel, C. H. et al. GISTIC2.0 facilitates sensitive and confident localization of the targets of focal somatic copy-number alteration in human cancers. Genome Biol. 12, R41 (2011).

9. Mroz, E. A. \& Rocco, J. W. MATH, a novel measure of intratumor genetic heterogeneity, is high in poor-outcome classes of head and neck squamous cell carcinoma. Oral. Oncol. 49, 211-215 (2013).

10. Forbes, S. A. et al. COSMIC: Somatic cancer genetics at high-resolution. Nucleic Acids Res. 45, D777-D783 (2017).

11. Lawrence, M. S. et al. Mutational heterogeneity in cancer and the search for new cancer-associated genes. Nature 499, 214-218 (2013).

12. Hanisch, F.-G. O-Glycosylation of the mucin type. Biol. Chem. 382, 143-149 (2001).

13. Anderson, S. M., Rudolph, M. C., McManaman, J. L. \& Neville, M. C. Key stages in mammary gland development. Secretory activation in the mammary gland: it's not just about milk protein synthesis! Breast Cancer Res. 9, 204 (2007).

14. Alexandrov, L. B. et al. Signatures of mutational processes in human cancer. Nature 500, 415-421 (2013).

15. Jiricny, J. The multifaceted mismatch-repair system. Nat. Rev. Mol. Cell Biol. 7, 335-346 (2006).

16. Mukhopadhyay, P. et al. Mucins in the pathogenesis of breast cancer: implications in diagnosis, prognosis and therapy. Biochim. Biophys. Acta 1815, 224-240 (2011).

17. Hollingsworth, M. A. \& Swanson, B. J. Mucins in cancer: protection and control of the cell surface. Nat. Rev. Cancer 4, 45-60 (2004).

18. Alexandrov, L. B. et al. Clock-like mutational processes in human somatic cells Nat. Genet. 47, 1402-1407 (2015).

19. Davies, H. et al. Whole-genome sequencing reveals breast cancers with mismatch repair deficiency. Cancer Res. https://doi.org/10.1158/0008-5472.CAN-17-1083 (2017).

20. Le, D. T. et al. PD-1 blockade in tumors with mismatch-repair deficiency. N. Engl. J. Med. 372, 2509-2520 (2015).

21. Rizvi, N. A. et al. Mutational landscape determines sensitivity to PD-1 blockade in non-small cell lung cancer. Science 348, 124-128 (2015).

22. Emens, L. A. Breast cancer immunotherapy: facts and hopes. Clin. Cancer Res. 3001, 2017, https://doi.org/10.1158/1078-0432.CCR-16-3001 (2017).

23. Huh, S. J. et al. Age and pregnancy-associated dna methylation changes in mammary epithelial cells. Stem Cell Rep. 4, 297-311 (2015).

24. Coates, A. S. et al. Tailoring therapies-improving the management of early breast cancer: St Gallen International Expert Consensus on the Primary Therapy of Early Breast Cancer 2015. Ann. Oncol. 26, 1533-1546 (2015).

25. TCGA. Comprehensive molecular portraits of human breast tumours. Nature 487, 61-70 (2012).

26. Rosenthal, R., McGranahan, N., Herrero, J., Taylor, B. S. \& Swanton, C. deconstructSigs: delineating mutational processes in single tumors distinguishes DNA repair deficiencies and patterns of carcinoma evolution. Genome Biol. 17, 31 (2016).

27. Benjamini, Y. \& Hochberg, Y. Controlling the false discovery rate: a practical and powerful approach to multiple testing. J. R. Stat. Soc. B 57, 289-300 (1995).

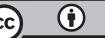
adaptation, distribution and reproduction in any medium or format, as long as you give appropriate credit to the original author(s) and the source, provide a link to the Creative Commons license, and indicate if changes were made. The images or other third party material in this article are included in the article's Creative Commons license, unless indicated otherwise in a credit line to the material. If material is not included in the article's Creative Commons license and your intended use is not permitted by statutory regulation or exceeds the permitted use, you will need to obtain permission directly from the copyright holder. To view a copy of this license, visit http://creativecommons. org/licenses/by/4.0/.

(c) The Author(s) 2018 